\title{
Gastric Outlet Obstruction Due to Cholecysto-Gastric Fistula: Bouveret's Syndrome
}

\author{
Savas Sezik ${ }^{1}$, Mustafa Sever ${ }^{1, \star}$, Nezih Kavak ${ }^{2}$, \\ Ahmet Burak Erdem ${ }^{3}$
}

\author{
${ }^{1}$ Department of Emergency Medicine, \\ Tepecik Educational and Research \\ Hospital, Izmir, Turkey \\ ${ }^{2}$ Department of Emergency Medicine, \\ Yildirim Beyazit Diskapi Educational and \\ Research Hospital, Ankara, Turkey \\ ${ }^{3}$ Department of Emergency Medicine, \\ Ankara Numune Educational and \\ Research Hospital, Ankara, Turkey

\section{*Correspondence} \\ adanasever@yahoo.com \\ (Mustafa Sever)
}

\begin{abstract}
Bouveret's syndrome is defined as gastrointestinal obstruction due to an impacted gallstone secondary to biliary-enteric fistula. This is often observed with nonspecific symptoms such as epigastric pain, nausea, and vomiting. The diagnosis is made by visualization of pneumobilia, gastric outlet obstruction and ectopic gallstone. In this report, we discussed an elderly patient who was admitted to the emergency department with abdominal pain and vomiting without any signs of bowel obstruction in plain x-ray and ultrasound imaging. We aimed to remind the importance of clinical suspicion and the diagnostic value of computed abdominal tomography for emergency physicians.
\end{abstract}

\section{Keywords}

Abdominal pain, Bouveret's syndrome, Computed tomography, Elderly, Emergency department

\section{Introduction}

Bilio-enteric fistulas are rare complications of gallstones, frequently following an episode of acute cholecystitis. The incidence of biliary fistulas during acute cholecystitis varies between $3-5 \%$ [1]. There are two types of fistulas; (1) between the gallbladder and the gastrointestinal tract and (2) between the common bile duct and the gastrointestinal tract $[2,3]$. The most common type, the cholecysto-duodenal tract fistula, was evident in $61 \%$ of cases. Cholecysto-colic, cholecysto-gastric, choledocho-douodenal and other fistulas are rarer $(11 \%, 4 \%, 13 \%$, and $11 \%$, respectively) [4]. Only $7-10 \%$ of biliary fistulas cause gallstone ileus due to the migration of the gallstone through the fistula and the gallstone can be obstructed anywhere in the gastrointestinal tract [2]. The most common localization is the terminal ileum and ileocecal valve $(70 \%)[2,3]$. The mechanical intestinal obstructions due to gallstones are $1-3 \%$ of all intestinal obstructions [5].

\section{Case Report}

A 71-year-old female was admitted to the emergency department with abdominal pain and vomiting, up to 1 week. She had hypertension and hyperlipidemia. Vital signs were normal except 153/95 mmHg blood pressure and tenderness on the right upper quadrant. There is no rebound tenderness. Serum liver enzymes, bilirubine, electrolytes, creatinine, and urea levels were in normal range. Complete blood count revealed mild leukocytosis. (13.5 k/ul; normal range: $4-10 \mathrm{k} / \mathrm{ul})$. Abdominal and lung radiographies were interpreted as normal (Fig. 1) and ultrasound was suboptimal due to gas interposition. Intravenous contrastenhanced abdominal computerized tomography (CT) was performed. In the $\mathrm{CT}$, it was revealed that there was pericholecystic fluid around the gallbladder and gallbladder wall was thickened and there was air in the liver, common bile duct and the gallbladder (pneumobilia) as well as a gallstone in the gastric antrum $(17 \times 13 \mathrm{~mm})$ (Fig. 2). In the endoscopic imaging, it was observed that the gallstone had impacted to the pyloric passage (Fig. 3). The gallstone could not be removed by endoscopic intervention therefore the patient was admitted for operation. Gastrostomy and extraction of the gallstones were performed and she was discharged seventeen days after admission without any complications.

\section{Discussion}

This syndrome was primarily observed in the sixth or seventh decade of patients and especially females [2-6]. Older age, female gender, gallstones larger than $2.5 \mathrm{~cm}$ and changed gastrointestinal morphology due to surgery are the 4 major risk factors for developing this syndrome 


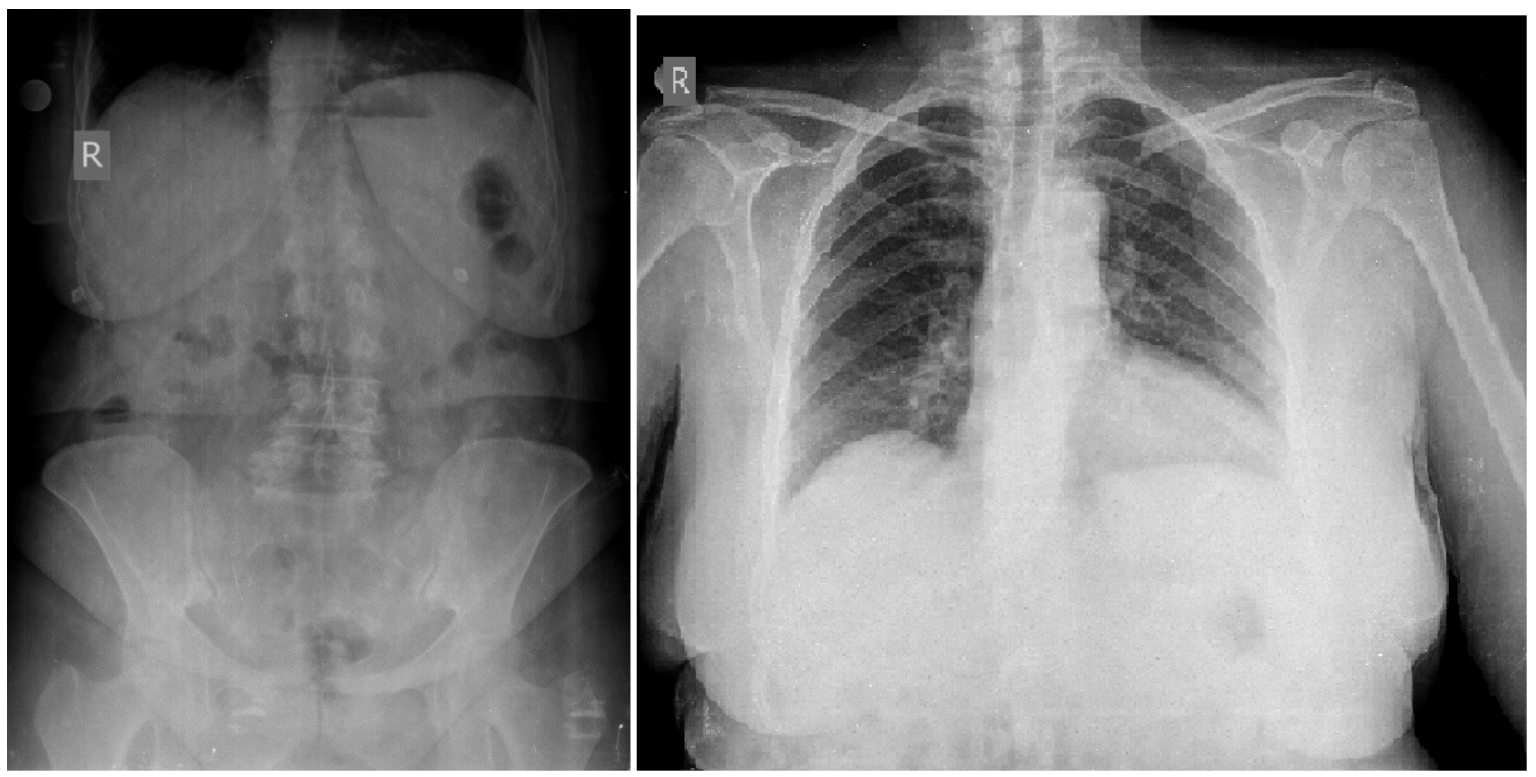

F I G U RE 1. Plain abdominal and AP lung x-ray graphies.

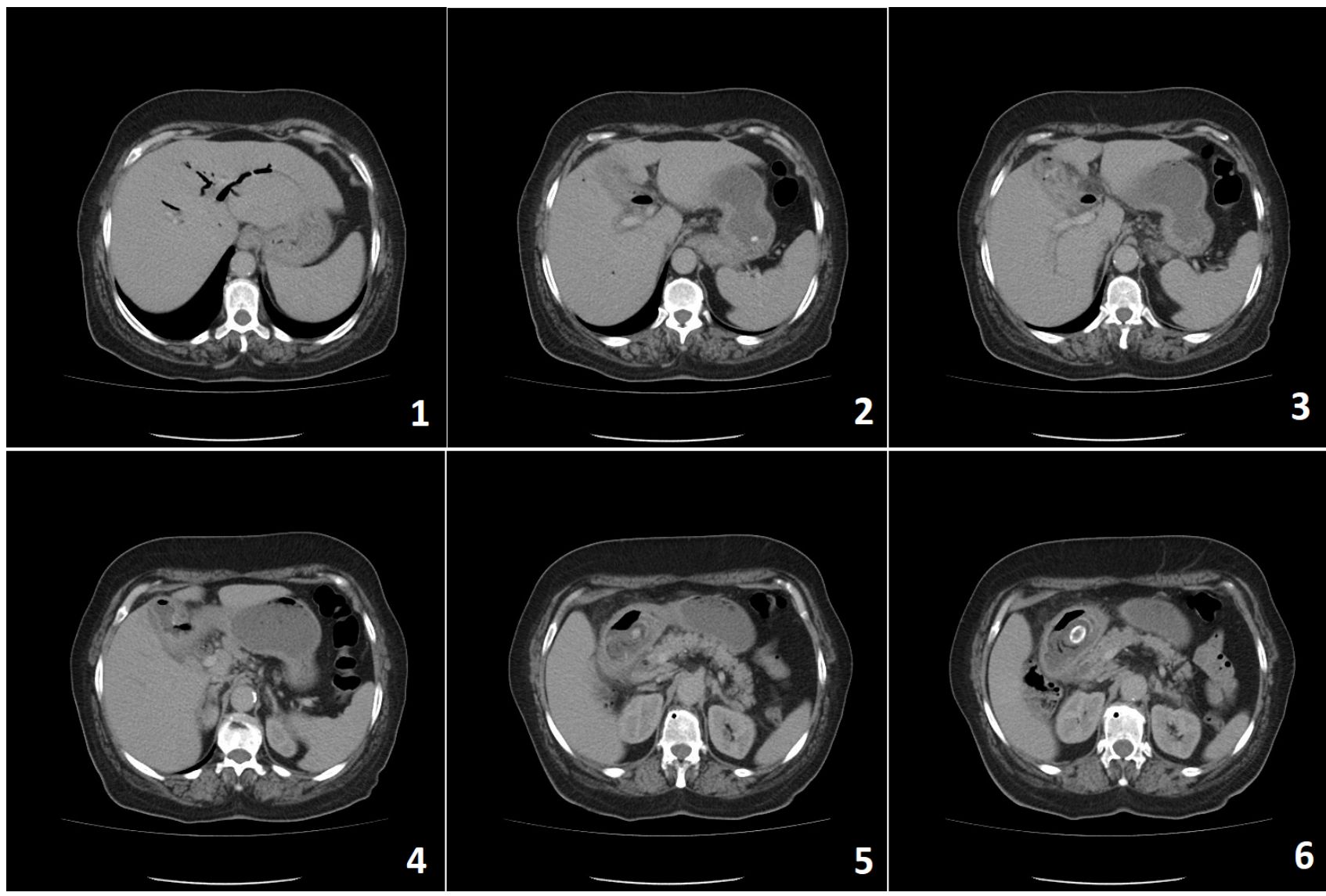

F I G U R E 2. IV contrast-enhanced abdominal computed tomography.

[6]. The signs and symptoms are often nonspecific and not different from mechanical bowel obstruction due to other etiologies [4]. Yakan S. et al [7] reported 12 cases of gallstone ileus; the most commonly observed symptoms were abdominal pain (100\%), vomiting (92\%), abdominal distension $(67 \%)$, constipation $(33 \%)$, fever $(17 \%)$ and jaundice [8] (8\%). Gastric or duodenal obstruction may not reveal abdominal distention and air-fluid levels in an abdominal x-ray, however gastric dilatation may be observed.

The ultrasonography and abdominal X-ray may help the diagnosis of Bouveret's syndrome. Ultrasonography is the first choice for assessing gallbladder wall thickness and pneumobilia [2-7]. The Rigler's triad (pneumobilia, small 

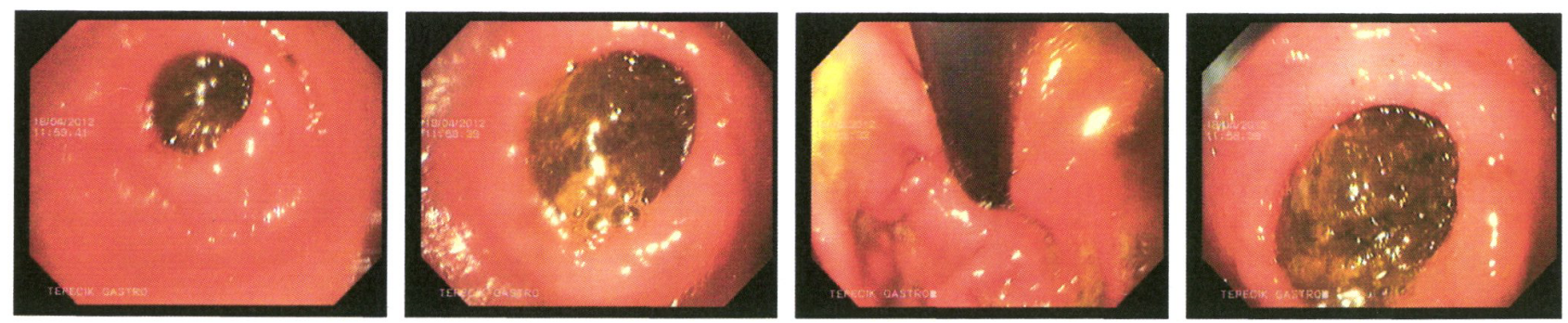

F I G U R E 3. Endoscopic imaging.

bowel obstruction and gallstone) was observed with plain films in $15 \%$ of patients, $11 \%$ with US and $78 \%$ with CT. Magnetic Resonance Cholangio-Pancreatography may be a useful tool for detecting impacted gallstone and the fistula [8].

Endoscopic stone removal using mechanical or laser lithotripsy is the first treatment choice. The success rate of extracting the stone with endoscopy was reported as only 10 - 40\% [7-9]. Noninvasive treatments such as intracorporeal electrohydraulic lithotripsy and extracorporeal shock wave lithotripsy should be considered [8]. Surgery is indicated in particular conditions such as stone impaction in the fistula, stone compression to the duodenal wall, gastrointestinal hemorrhage and failure by endoscopic removal $[1,2,4,6,9]$.

It is well known that Bouveret's Syndrome causes significant morbidity and mortality. The diagnosis is often delayed, with a resulting mortality rate of up to 20 [10]. Emergency physicians should be aware of Bouveret's syndrome in patients presenting with pneumobilia, gallbladder wall thickness and small bowel obstruction.

\section{LEARNING POINTS}

Bouveret's syndrome is a rare and important clinical entity and it may also present itself with nonspecific symptoms. Conventional radiography and ultrasonography may show no benefits. This case report shows the benefit of computerized tomography in unexplained abdominal pains with nausea and vomiting and without an ileus such as variant Bouveret's syndrome.

\section{ACKNOWLEDGEMENTS}

We would like to thank Okan Sayan for English reduction in writing this manuscript.

\section{CONFLICT OF INTEREST}

The authors' declared that there is no conflict of interest.

\section{ETHICAL APPROVAL}

Not applicable.

\section{FUNDING INFORMATION}

This manuscript did not receive any specific grant from any funding agency in the public, commercial, or not-for-profit sectors.

\section{INFORMED CONSENT}

Informed consent was observed verbally for the present manuscript.

\section{HUMAN RIGHTS}

Our work does not infringe on any rights of others, including privacy rights, and intellectual property rights. There is no human rights violation in our manuscript.

\section{REFERENCES}

[1] Brennan GB, Rosenberg RD, Arora S. Bouveret Syndrome. Radiographics. 2004;24:1171-1175.

[2] Arioli D, Venturini I, Masetti M, et al. Intermittent gastric outlet obstruction due to a gallstone migrated through a cholecysto-gastric fistula: a new variant of Bouveret's syndrome. World J Gastroenterol. 2008; $14: 125-128$.

[3] Matur R, Yucel T, Gurdal SO, et al. Bouveret Sendromu: Safra Tasına Baglı Mide Çıkıs Obstruksiyonu. Ulus Travma Derg. 2002;8:179182.

[4] Petrowsky H., Pierre-A.C. "Biliary fistula, gallstone ileus and Mirizzi’s syndrome" In: Clavien P.A., Baillie J. Disease of the gallbladder and bile ducts: Diagnosis and treatment; 2006. p. 23948.

[5] Martínez Ramos D, Daroca José JM, Escrig S, et al. Gallstone ileus: management options and results on a series of 40 patients. Rev Esp Enferm Dig. 2009;101:117-124.

[6] Gajendran M, Muniraj T, Gelrud A. A challenging case of gastric outlet obstruction (Bouveret's syndrome): A case report. J Med Case Rep. 2011;4: 497.

[7] Yakan S, Engin Ö. Gallstone ileus as an unexpected complication of cholelithiasis: diagnostic difficulties and treatment. Ulus Travma Acil Cerrahi Derg. 2010;16:344-348.

[8] Pickhardt PJ, Friedland JA, Hruza DS, et al. CT, MR cholangiopancreatography, and endoscopy findings in Bouveret's syndrome. Am J Roentgenol. 2003;180:1033-1035.

[9] Doycheva I, Limaye A, Suman A, et al. Bouveret's syndrome: Case report and review of the literature. Gastroenterol Res Pract. 2009;2009:914-951.

[10] David Q.-H. Wang and Nezam H. Afdhal. Section VIII: Biliary Tract. Chapter 65: Gallstone Disease. In: Sleisenger and Fordtran's Gastrointestinal and Liver Disease. Pathophysiol- 
ogy/Diagnosis/Management. Eds.: Feldman M, Friedman LS, Brandt LJ. E-Book. 9th Ed. Saunders-Elsevier. Pp: 1089-1119.
How to cite this article: Savas Sezik, Mustafa Sever, Nezih Kavak, Ahmet Burak Erdem. Gastric Outlet Obstruction Due to Cholecysto-Gastric Fistula: Bouveret's Syndrome. Signa Vitae. 2021;17(1):209-212. doi:10.22514/sv.2020.16.0036. 